\title{
Hypoxia induces no change in cutaneous thresholds for warmth and cold sensation
}

\author{
U. L. Malanda · J. P. H. Reulen · W. H. M. Saris • \\ W. D. van Marken Lichtenbelt
}

Accepted: 10 March 2008 / Published online: 26 March 2008

(C) The Author(s) 2008

\begin{abstract}
Hypoxia can affect perception of temperature stimuli by impeding thermoregulation at a neural level. Whether this impact on the thermoregulatory response is solely due to affected thermoregulation is not clear, since reaction time may also be affected by hypoxia. Therefore, we studied the effect of hypoxia on thermal perception thresholds for warmth and cold. Thermal perception thresholds were determined in 11 healthy overweight adult males using two methods for small nerve fibre functioning: a reaction-time inclusive method of limits (MLI) and a reaction time exclusive method of levels (MLE). The subjects were measured under normoxic and hypoxic conditions using a cross-over design. Before the thermal threshold tests under hypoxic conditions were conducted, the subjects were acclimatized by staying 14 days overnight $(8 \mathrm{~h})$ in a hypoxic tent system (Colorado Altitude Training: 4,000 m). For normoxic measurements the same subjects were not acclimatized, but were used to sleep in the same tent system. Measurements were performed in the early morning in the tent. Normoxic MLI cold sensation threshold decreased significantly from $30.3 \pm 0.4$ (mean $\pm \mathrm{SD}$ ) to $29.9 \pm 0.7^{\circ} \mathrm{C}$ when exposed to hypoxia $(P<0.05)$. Similarly, mean normoxic MLI warm sensation threshold increased from $34.0 \pm 0.9$ to $34.5 \pm 1.1^{\circ} \mathrm{C}(P<0.05)$. MLE measured
\end{abstract}

U. L. Malanda · W. H. M. Saris .

W. D. van Marken Lichtenbelt ( $ه)$

Department of Human Biology, Nutrition and Toxicology

Research Institute Maastricht (NUTRIM), Maastricht University,

P.O. Box 616, 6200 MD Maastricht, The Netherlands

e-mail: Markenlichtenbelt@hb.unimaas.nl

J. P. H. Reulen

Department of Clinical Neurophysiology,

Maastricht University Hospital, P.O. Box 5800,

6202 AZ, Maastricht, The Netherlands threshold for cutaneous cold sensation was $31.4 \pm 0.4$ and $31.2 \pm 0.9^{\circ} \mathrm{C}$ under respectively normoxic and hypoxic conditions $(P>0.05)$. Neither was there a significant change in MLE warm threshold comparing normoxic $\left(32.8 \pm 0.9^{\circ} \mathrm{C}\right)$ with hypoxic condition $\left(32.9 \pm 1.0^{\circ} \mathrm{C}\right)$ $(P>0.05)$. Exposure to normobaric hypoxia induces slowing of neural activity in the sensor-to-effector pathway and does not affect cutaneous sensation threshold for either warmth or cold detection.

Keywords Thermoregulation · Thermal thresholds · Temperature sensitivity $\cdot$ Intermittent normobaric hypoxia

\section{Introduction}

Thermoregulatory responses in humans exposed to acute hypoxia have been studied extensively (Golja and Mekjavic 2003; Johnston et al. 1996). Most research on this topic has been focused on the one hand on thermal perception (Golja et al. 2004; Golja and Mekjavic 2003; Launay et al. 2006) and on the other on reaction-time (Mackintosh et al. 1988) both pleading for further research on the cause.

Hypoxia is suggested to affect perception of temperature stimuli by impeding thermoregulation at a neural level and may influence the zone of thermal comfort. Whether this impact on the thermoregulatory response is solely due to affected thermal regulation can be argued since hypoxia may also affect reaction-time (Fowler and Prlic 1995; Mackintosh et al. 1988). Recently acclimation to intermittent hypoxia has been described to increase the sensitivity of the thermoregulatory system and thereby to modify thermoregulatory responses at sea level (Launay et al. 2006). This could lead to a disturbance in the cutaneous perception of warm and cold stimuli. Golja et al. (2004) investigated 
the effects of hypoxic exposure on the perception of cutaneous warm and cold stimuli and suggested that cold sensation is affected as a result of exposure to hypoxia. This assumption was assessed with a cutaneous threshold thermal testing system positioned at the feet while subjects where either under hypocapnic hypoxia or normocapnic hypoxia conditions. Previous studies have demonstrated that hypoxic exposure could lead to a decrease in neural function resulting in a loss in cognitive function (Fowler et al. 1994; Kennedy et al. 1989) and an increase in reaction time (Fowler et al. 1994).

The aim for this study was to assess the effect of nocturnal induced normobaric intermittent hypoxia on thermal perception thresholds (warm and cold) using a reaction time inclusive (Limits) and a reaction time exclusive (Levels) method for small nerve functioning in men. We hypothesize that hypoxia induces slowing of neural activity in the sensor-to-effector pathway and does not affect the sensation threshold for detection of either warm or cold. As a consequence of this hypothesis the results of Levels testing must be unaffected by hypoxia while the corresponding Limits values are expected to be significantly changed.

\section{Methods}

\section{Subjects and materials}

Eleven healthy, non-smoking overweight males with an average (SD) age of 44 (7) years, a height of $180(7) \mathrm{cm}$, a body mass of $104.9(16.1) \mathrm{kg}$ and a body mass index of $32.2(3.1) \mathrm{kg} / \mathrm{m}^{2}$, volunteered for the study (Table 1). As this study was part of a larger investigation on the effect of hypoxia in overweight men, all subjects were overweight. To exclude heart disease all subjects had a screening ECG before giving their informed consent. The medical ethical committee of the Maastricht University approved the study. All subjects signed a written informed consent for the study protocol.

Nocturnal hypoxia is induced using a tent with a specially designed controlled air infiltration rate. This enclosure is coupled with a molecular sieve that produces hypoxic air. A compressor forces normoxic air through the molecular sieve producing a controlled level of hypoxia.

Table 1 Subject characteristics $(n=11)$

\begin{tabular}{lll}
\hline$n=11$ & Mean (SD) & Range \\
\hline Age (years) & $44(7)$ & $35-55$ \\
Height $(\mathrm{cm})$ & $180(7)$ & $169-196$ \\
Body mass $(\mathrm{kg})$ & $104.9(16.1)$ & $85.9-139.7$ \\
BMI $\left(\mathrm{kg} / \mathrm{m}^{2}\right)$ & $32.2(3.1)$ & $28.0-36.9$ \\
\hline
\end{tabular}

Airflow through the tent is sufficient to maintain high air quality and safe $\mathrm{CO}_{2}$ levels while simulating altitudes up to $4,575 \mathrm{~m}$ and is therefore widely used in altitude training. The tent used in this study was $2.1 \times 2.3 \times 1.8 \mathrm{~m}$ and incorporates an oxygen sensor and hypoxic alarm (Wilber 2001). Before the thermal threshold tests were conducted, the subjects stayed overnight $(8 \mathrm{~h})$ in a hypoxic tent system (Colorado Altitude Training) under normoxic $(300 \mathrm{~m})$ and hypoxic $(4,000-4,200 \mathrm{~m})$ conditions at the department of Human Biology at the Maastricht University. The study had a crossover design with a normoxic and a hypoxic period, preceeding the measurements in the laboratory. Each subject was measured after a normoxic period of at least 14 days preceeding the normoxic measurements, and after a 14 days hypoxic period before the hypoxic measurements. To avoid any tent effects all volunteers spent at least three nights at home sleeping under normoxic conditions in a fully operational tent before normoxic measurements. To prevent symptoms of acute mountain sickness subjects where acclimatized to hypoxia corresponding to $4,200 \mathrm{~m}$. This stair step build-up was done in 5 days according to the so-called 300-m rule. After this period subjects were exposed to hypoxia at home every night for 14 consecutive days before the laboratory measurements commenced. The evening before the measurements participants came to the institute at 21:30 h. To get acquainted with the temperature threshold test procedures each subject performed a testing session consisting of a limits and a levels sequence in the evening (see below). Skin temperatures were measured according to Ramanathan (1964) by means of wireless thermo telemetry sensors (iButton ${ }^{\circledR}$ ) (van Marken Lichtenbelt et al. 2006). Core body temperature was measured by means of temperature telemetry using commercially available medical grade capsules (Coretemp ${ }^{\mathrm{TM}}$ 2000). After placing the temperature sensors, subjects got ready to enter the tent.

All measurements were performed inside the hypoxic tent under either normoxic or hypoxic conditions. Approximately $30 \mathrm{~min}$ after waking up in the morning subjects where summoned to sit at the foot end of the bed while temperature thresholds were measured at the palmer thenar of the contra lateral wrist, as opposed to right- or left-handedness. The researcher entered the tent via a lock system ensuring a constant $\mathrm{O}_{2}$ level. The tests were performed between 7:00 a.m. and 7:30 a.m. The tests were performed according to Reulen et al. (2003) using a commercially available computerized thermo-stimulator (Medoc TSA $2001^{\circledR}$, Ramat Yishai, Israel) operating on the Peltier principle. The thermode (active area $32 \times 32 \mathrm{~mm}^{2}$ ) was strapped to the palmar thenar, distal of the carpometacarpal joint using a fixation belt. The free hand was used to press a mouse button either when warm or cold sensation was perceived or a "yes/no" answer had to be given. Thermode 
adaptation temperature and thus starting baseline temperature was always set to $32^{\circ} \mathrm{C}$.

\section{Method description}

Thermal perception thresholds were determined using two methods for testing of small nerve fibre functioning: the reaction-time inclusive method of limits (MLI) and the reaction time exclusive method of levels (MLE) as described by Claus et al. (1987).

\section{Methods of limits (MLI)}

The MLI is based on an increasing or decreasing stimulus temperature with a constant rate of change $\left(1^{\circ} \mathrm{C} / \mathrm{s}\right)$ starting from a preset adaptation temperature. As soon as warm or cold sensation is perceived the subject stops the administered stimulus by pushing a button thereby resulting in a slightly higher (increasing warm stimulus) or lower (decreasing cold stimulus) perceived threshold temperature because of the inherently "built-in" reaction time (Fig. 1a). A warm or cold sequence consists of five temperature stimuli and final MLI threshold is the mean of these five values. In advance of each sequence two training stimuli were administered. A complete MLI protocol started with seven cold stimuli ( 2 training, 5 measurement) followed by seven warm stimuli ( 2 training, 5 measurement). The inter-stimulus interval varied at random between 4 and $6 \mathrm{~s}$ and the change rate was set at $1^{\circ} \mathrm{C} / \mathrm{s}$ with a $10^{\circ} \mathrm{C} / \mathrm{s}$ return rate to baseline upon pushing the reaction button. To alert the subject an auditory warning signal was given directly before onset of each stimulus.

\section{Methods of levels (MLE)}

MLE is characterised by confirming or denying a well-defined temperature change. Starting from $32^{\circ} \mathrm{C}$ temperature rises (warm sensation) or decreases (cold sensation) with a $2^{\circ} \mathrm{C}$ step (rate of change $1^{\circ} \mathrm{C} / \mathrm{s}$ ). Based on the subjects answer ("yes" or "no" sensation) the ${ }^{\circ} \mathrm{C}$ amplitude of the following temperature step is doubled ("no" answer) or halved ("yes" answer) until a minimal perceptive criterion is established. In this "yes/no" procedure post-stimulus speed of reaction and by that reaction time does not play a role. By doing so a complete MLE test consists of several single stimuli resulting in a finally acquired reaction-time free temperature threshold. Anticipation or prediction of stimuli is prevented by random inclusion of "dummies" (no temperature change after the auditory signal) and combining two separate sequences of levels stimuli in a single test sequence. In this study levels thresholds were determined by applying temperature stimuli directly after an auditory cue (change rate $1^{\circ} \mathrm{C} / \mathrm{s}$ ) (Fig. 1b). Similar to the MLI, cold
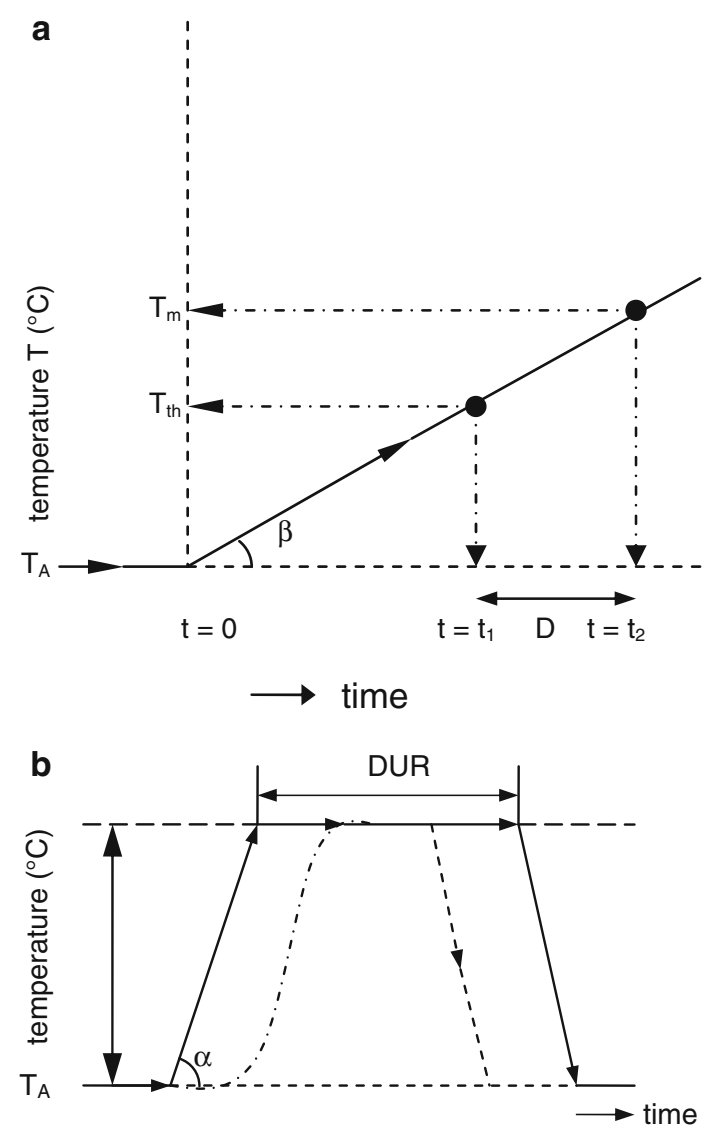

Fig. 1 a Linear threshold-crossing model for warm sensation and cold sensation; temperature (T) as a function of time for a stimulus starting from $T_{\mathrm{A}}$ at $t=0$ with a constant $V_{T}\left(\tan \beta^{\circ} \mathrm{C} / \mathrm{s}\right)$. Sensation occurs when at $t=t_{1}$, T cresses threshold value $T_{\text {th. }}$ During the reaction time delay (D), temperature still increases until the subject halts the stimulus $\left(T=T_{m}\right)$ at $t=t_{2}$. b Amplitude-time profile of basic MLE stimulus configuration. The stimulus starts with a temperature increase with fixed $V_{t}(\tan \alpha)$; as soon as maximal stimulus temperature $\left(T_{\mathrm{A}}+\Delta \mathrm{T}\right)$ is reached it remains at that value for a fixed period (DUR). After that temperature decreases back to $T_{\mathrm{A}}\left(V_{t}=\tan \beta\right)$. Tan $\alpha$ is chosen $1{ }^{\circ} \mathrm{C} / \mathrm{s}$ and return to $T_{\mathrm{A}}(\tan \beta)$ is set to $4^{\circ} \mathrm{C} / \mathrm{s}$. After return to $T_{\mathrm{A}}$, the participant has to respond 'yes' or 'no' sensation. To prevent unwanted skin energy dissipation, cooling down starts immediately after the participant's response (right dashed lines). Source: (Reulen et al. 2003)

sensation sequence preceded warm sensation. Return to adaptation temperature $\left(32^{\circ} \mathrm{C}\right)$ started as soon as participant responded "yes" or "no" (return rate $4^{\circ} \mathrm{C} / \mathrm{s}$ ) (Fig. 1b). The inter-stimulus interval was randomized between 4 and $6 \mathrm{~s}$ and the minimal perceptive criterion was set to $0.1^{\circ} \mathrm{C}$. Final MLE threshold for either cold or warm sensation was considered the mean of the last "yes" and "no" answered temperature step value.

The complete experimental protocol consisted of 2 MLI and 2 MLE series. It started with one series of limits (MLI 1), followed by a combination of two separate sequences of levels (MLE 1 and MLE 2) and ended again with a series of limits (MLI 2). Final limits thresholds (cold and warm) were considered the mean of MLI 1 and MLI 2 thresholds. 
Final levels thresholds (cold and warm) were considered the average of MLE 1 and MLE 2 thresholds.

Data analyses

Students $t$ tests and Wilcoxon signed rank tests (Statview $\mathrm{SE}^{+}$Graphics ${ }^{\mathrm{TM}}$ ) were used to analyze differences between pre and post measurements for final thermal thresholds (MLI and MLE), skin and core temperatures. For all analyses a statistical significance level of $P<0.05$ is used.

\section{Results}

All but one subject stayed at home at an altitude corresponding to 4,000-4,200 $\mathrm{m}\left(\mathrm{PO}_{2}: 12.5-11.9 \mathrm{kPa}\right)$. One subject experienced mild symptoms of acute mountain sickness and fulfilled his nocturnal stay at 3,300 m. In total an average of 7:20 h (0:34) (range: 6:26-8:11) was spent each night in the tent at home under hypoxia.

During the stay at the laboratory, no sleeping problems, nausea or sickness due to hypoxia were reported. Mean exposure to nocturnal hypoxia at the lab was 7:06 $\mathrm{h}(0: 27)$ (range: 6:23-8:00). Room temperature was kept constant at $21^{\circ} \mathrm{C}$ on all measuring days. Mean tent temperature was not significant different between normoxia and hypoxia [21.3 $(0.5)$ and $21.6(0.8)^{\circ} \mathrm{C}$ respectively; $\left.P>0.05\right]$.

Average skin temperature showed no significant differences between normoxia $\left[33.4(0.7)^{\circ} \mathrm{C}\right]$ and hypoxia [33.6 $\left.(0.6)^{\circ} \mathrm{C}\right](P>0.05)$. Core temperature registration failed in one subject. Mean body core temperature in normoxic condition $\left[36.5(0.1)^{\circ} \mathrm{C}\right]$ was not significant different from hypoxia $\left[36.6(0.2)^{\circ} \mathrm{C}\right](P>0.05)$.

The average normoxic MLI cold sensation threshold decreased significantly from $30.3(0.4)$ to $29.9(0.7)^{\circ} \mathrm{C}$ under hypoxic exposure $(P<0.05$, Table 2$)$. Mean MLI warm sensation threshold significantly increased from 34.0 $(0.9)^{\circ} \mathrm{C}$ under normoxia to $34.5(1.1)^{\circ} \mathrm{C}$ under hypoxia $(P<0.05$, Table 2$)$. The mean MLE cold sensation threshold was respectively $31.4(0.4)$ and $31.2(0.9)^{\circ} \mathrm{C}$ for either normoxia and hypoxia (Table 2) and was not significantly different between both conditions $(P=0.22)$. Neither were significant changes in MLE warm sensation threshold

Table 2 Cutaneous thermoregulatory thresholds $\left({ }^{\circ} \mathrm{C}\right)$ during normoxic and hypoxic conditions

\begin{tabular}{llllll}
\hline Factor & $N$ & Normoxia (SD) & Hypoxia (SD) & $t$ test & Wilcoxon \\
\hline MLI cold & 11 & $30.3(0.4)$ & $29.9(0.7)$ & 0.029 & 0.026 \\
MLI warm & 11 & $34.0(0.9)$ & $34.5(1.1)$ & 0.024 & 0.033 \\
MLE cold & 11 & $31.4(0.4)$ & $31.2(0.9)$ & 0.22 & 0.21 \\
MLE warm & 11 & $32.8(0.9)$ & $32.9(1.0)$ & 0.26 & 0.25 \\
\hline
\end{tabular}

observed under normoxic $\left[32.8(0.9)^{\circ} \mathrm{C}\right]$ compared to hypoxic conditions $\left[32.9(1.0)^{\circ} \mathrm{C}\right.$, Table 2$](P=0.26)$.

Except for three outliers (marked $*$ in Figs. 2b, 3a, b), all individual normoxia and hypoxia thresholds correspond to normative data for thermal testing at this body location as published by Yarnitsky and Sprecher (1994). These outliers did not influence the general findings of our study for correct interpretation of the results above.

\section{Discussion}

In the present study we compared normoxic temperature sensitivity thresholds in non-acclimatized subjects with hypoxic temperature sensitivity thresholds in the same but hypoxia acclimatized subjects. The results show that cold and warm cutaneous temperature threshold sensitivities are not affected by exposure to normobaric hypoxia in altitude acclimatized, overweight subjects. This is based on the finding of unchanged cold and warm temperature thresh-
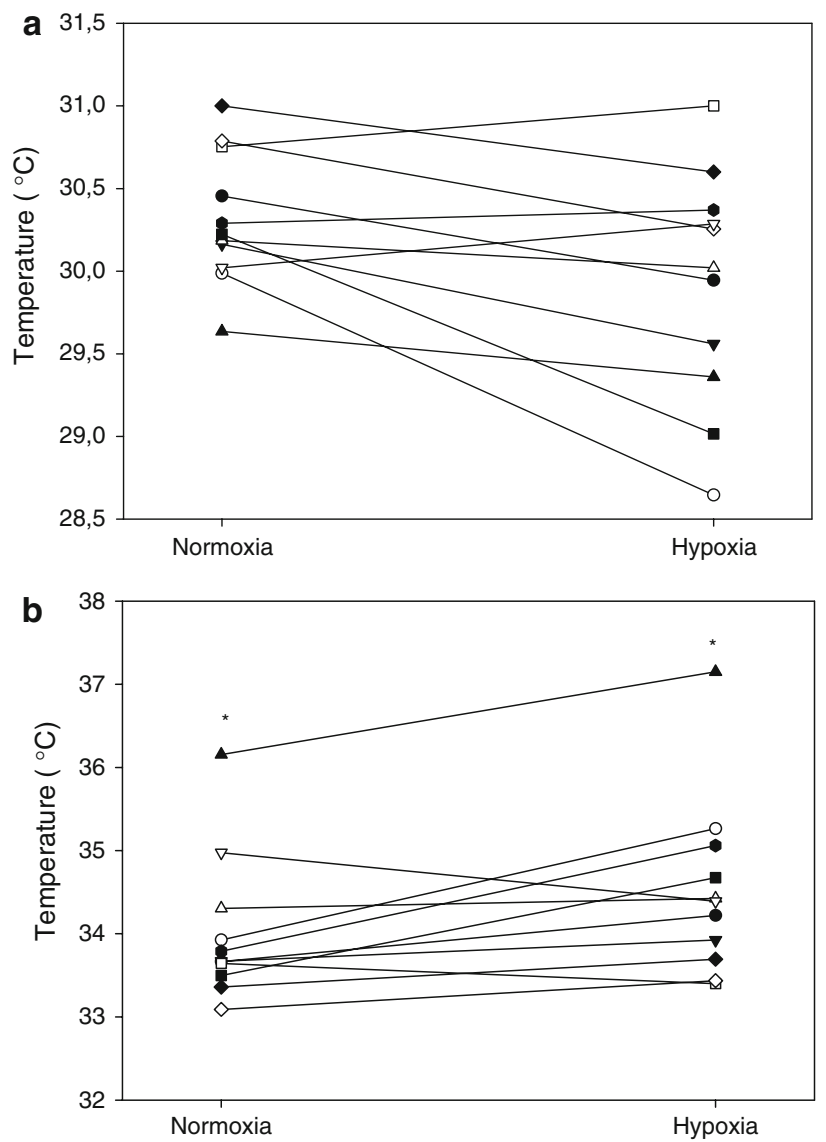

Fig. 2 a Average threshold for the sensation of cold during normoxia and hypoxia per subject. Measured with the reaction time inclusive method of limits. b Average threshold for the sensation of warmth during normoxia and hypoxia per subject. Measured with the reaction time inclusive method of limits. Asterisk threshold outside normative data (Yarnitsky and Sprecher 1994) 

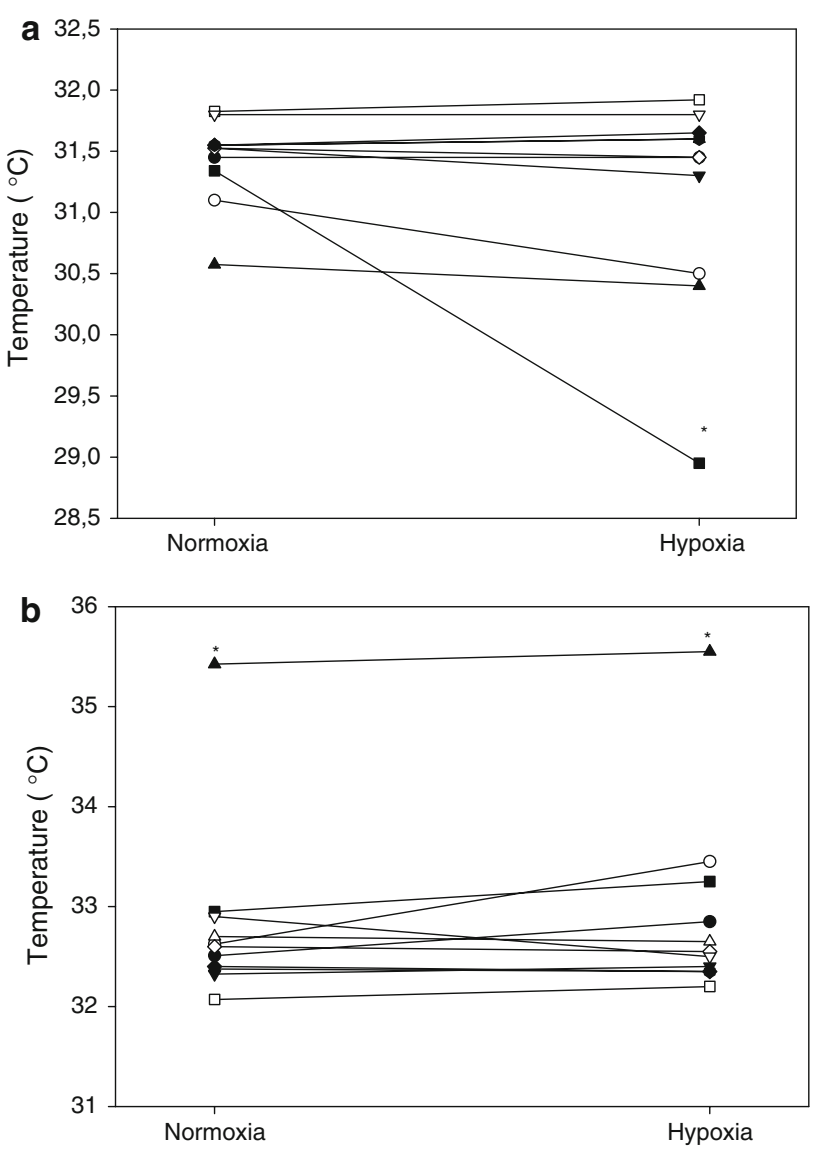

Fig. 3 a Average threshold for the sensation of cold during normoxia and hypoxia per subject. Measured with the reaction time exclusive method of levels. Asterisk threshold outside normative data (Yarnitsky and Sprecher 1994). b Average threshold for the sensation of warmth during normoxia and hypoxia per subject. Measured with the reaction time exclusive method of levels. Asterisk threshold outside normative data (Yarnitsky and Sprecher 1994)

olds at the palmar thenar of the hand using the method of Levels. The increase in processing time in the sensor-toeffector pathway, including reaction time, becomes apparent from increased MLI measured thresholds for warm and cold.

In a study in which the perception of cold and warm cutaneous stimuli under hypoxia and hypoxia induced hypocapnia was examined, Golja et al. (2004) have reported a decrease in cutaneous thermal sensation for cold, but not for warmth, in humans after inspiring a mixture containing $10 \%$ oxygen. These results where obtained by determining cutaneous thresholds for thermal sensation on the toe using an MLE alike levels method. Their finding of an unchanged warmth threshold agrees with our findings while the increased threshold for cold disagrees with our data. However, there are major methodological differences between both studies. A difference was the level of acclimatization. While Golja et al. (2004) measured under nonacclimatized conditions, the volunteers in the present study were acclimatised prior to the tests by 14 days nocturnal intermittent hypoxia. Acclimatization thus can be one reason for the discrepancy for cold sensation between Golja et al. 2004 and our study. Another difference concerns the method and apparatus used for threshold determination, which were different from ours. Stimulus site was also different (hand versus toe). In Golja's study warm threshold of the toes was measurable in a limited number of subjects. In our study all subjects responded to warm and cold stimulation of the hand.

Our study thus shows that the thermal thresholds, determined by the MLE, revealed no differences between normoxia and hypoxia. The altered thermal sensation as revealed by the MLI indicate that the hypoxic effect on cutaneous thermal thresholds may be a result of lowered neural activity and/or delayed signal conduction. This reflects the view that synaptic function is the first neural process to become affected during oxygen depletion (Astrup 1982). So the functioning of the "thermal sensitivity system" does not seem to be affected but processing speed is lowered. Since thermal thresholds are not changed it is unlikely that the zone of thermal comfort is affected by hypoxia under normobaric circumstances.

One may argue that during hypoxia no changes in thermal MLE sensation were observed, not because no effects exist on thermal sensation, but because other factors, such as a learning effect, may have interfered with the results. Golja and Mekjavic (2003) reported a decrease over consecutive trials in warmth sensation threshold using a levels alike method (Fowler and Taylor 1987). The warm threshold decreased with repeated testing and stabilised after the third trial, indicating a learning effect. In our study several arguments do not favour the view of a learning effect as the source of our finding of unchanged warm and cold MLE thresholds. The MLE method, using exactly the same Medoc TSA system with similar protocols as in our study, has been evaluated extensively (Yarnitsky and Sprecher 1994; Kemler et al. 2000). None of these studies, report significant intersession differences in MLE warm and cold thresholds measured at the wrist position between a first and a second session. In our laboratory (Kemler et al. 2000; Reulen etal. 2003) and in our patient investigations (Hoitsma et al. 2003) always one or more training session(s) were done before actual MLE threshold determination. As a consequence of the rather simply to perform procedure, post-trainings session MLE threshold did not depend on trial number. Furthermore, the effect found in our study was not warmth specific, as it was in Golja's (Golja and Mekjavic 2003) study but was comparable for warm and cold sensation thresholds. To avoid training effects, as mentioned in the method section, each subject performed a complete testing session the evening before the actual recording session took place. Finally, the study had a 
cross over design regarding their normo- or hypoxic measurements: four subjects started with the normoxic period and measurment versus seven who started with the hypoxic condition. This minimizes a possible systematic effect on the results.

Under normal conditions thermal stimuli are considered warm or cold depending on the cortical integration of afferent sensory information (Cabanac et al. 1969) mediated by relatively fast $(5-30 \mathrm{~m} / \mathrm{s})$ conducting $\mathrm{A} \delta$-fibers (cold perception) and slow (1-2.5 m/s) conducting C-fibers (warm perception) (Reulen et al. 2003). Since the MLI results did not differ between cold and warm tests, hypoxia did not seem to affect thermal perception of warm or cold differently. This might indicate that there may be no distinction in hypoxic impact on $\mathrm{A} \delta$ and $\mathrm{C}$-fiber function, and cortical integration of its afferent sensory information. Possibly, with respect to the reaction time, the efferent part of the trajectory is involved, but this needs further exploration.

Most information on thermoregulation and other physiological parameters is collected from hypobaric chamber studies and mountain expeditions. However, it is known that using 14 days of intermittent isobaric hypoxia also results in altitude acclimatization (Katayama et al. 2004). Our study shows that the body temperature profile, as reflected by core body and average skin temperature, did not significantly differ between normoxia and hypoxia. Since room temperature was kept constant and tent temperature did not differ per situation, no changes in body temperature distribution were expected. In the tent overnight $\mathrm{CO}_{2}$ accumulation occurred to a level of $0.3 \% \mathrm{CO}_{2}$. This is far below known measurable effects on thermoregulation. Calculation of mean skin temperature is better using more measurements sites, although a four-point mean is recommended where simplicity of the technique becomes important (Mitchell and Wyndham 1969). Keeping in mind the limitation of the four-point mean skin temperature, the results of this normobaric hypoxic study on core body and average skin temperature suggest that hypoxia per se does not affect the body temperature profile.

Exposure to hypoxia could lead to reduced attentional performance (Bonnon et al. 1995, 2000). Determination of thermal perception thresholds requires not only the ability to discriminate between thermal warm versus cold thresholds but in this case also the maintenance of an attentional focus. In the experimental setting used in our study, the auditory cue upon start of temperature change was thought to optimise attention. Stimulus duration, from its start to the moment of pushing the reaction button, was maximally about $4 \mathrm{~s}$ (time between start from $32^{\circ} \mathrm{C}$ to reaching a maximal threshold of about 28 or $36^{\circ} \mathrm{C}$ for cold respectively warmth). Under the (unlikely) assumption that subjects were not able upon auditory cued stimulus onset to hold attention for about $4 \mathrm{~s}$, one cannot completely reject decreased attentional performance as a cause or at least as a part of the noticed increase in reaction time.

Mackintosh et al. (1988) found a strong positive correlation between "long" reaction times and altitude in subjects affected with acute mountain sickness. As all subjects in the present study were acclimatized to hypoxia none of them suffered from any acute mountain sickness symptoms during testing. Considering this, lassitude or other effects associated with acute mountain sickness that might be able to interfere with reaction time can be excluded and thus, the noticed increase in the sensor-to-effector pathway processing time might be a direct hypoxic effect.

As the present study was part of an investigation in the effects of hypoxia on overweight and obesity, all subjects were overweight. Although there is no evidence stating differences in thermoregulatory reactions, one cannot exclude that discrepancy in results might occur between overweight and non-overweight subjects.

Finally we were able to confirm the hypothesis posed in the introduction. Although the influence of attention on MLI results cannot be excluded, precautions were taken to minimise it. A question remains whether decreased attention also may be a manifestation of decreased neural activity and/or signal conduction speed due to hypoxia.

In conclusion, the results of this research offer strong evidence for decreased neural processing and/or decreased nerve conduction speed as an important cause for hypoxia induced effects on nervous system functioning.

Acknowledgments The authors would express their gratitude to Colorado Altitude Training for supplying several CAT hypoxic tent systems and specific technical assistance. Loek Wouters, Paul Schoffelen and Maaike Head are thanked for their overall assistance.

Open Access This article is distributed under the terms of the Creative Commons Attribution Noncommercial License which permits any noncommercial use, distribution, and reproduction in any medium, provided the original author(s) and source are credited.

\section{References}

Astrup J (1982) Energy-requiring cell functions in the ischemic brain. Their critical supply and possible inhibition in protective therapy. J Neurosurg 56:482-497

Bonnon M, Noel-Jorand MC, Therme P (1995) Psychological changes during altitude hypoxia. Aviat Space Environ Med 66:330335

Bonnon M, Noel-Jorand MC, Therme P (2000) Effects of different stay durations on attentional performance during two mountain expeditions. Aviat Space Environ Med 71:678-684

Cabanac M, Ramel P, Duclaux R, Joli M (1969) Indifference to pain and thermic comfort (2 cases). Presse Med 77:2053-2054

Claus D, Hilz MJ, Neundorfer B (1987) Methods of measurement of thermal thresholds. Acta Neurol Scand 76:288-296

Fowler B, Prlic H (1995) A comparison of visual and auditory reaction time and P300 latency thresholds to acute hypoxia. Aviat Space Environ Med 66:645-650 
Fowler B, Prlic H, Brabant M (1994) Acute hypoxia fails to influence two aspects of short-term memory: implications for the source of cognitive deficits. Aviat Space Environ Med 65:641-645

Fowler B, Taylor M (1987) The effects of hypoxia on reaction time and movement time components of a perceptual-motor task. Ergonomics 30:1475-1485

Golja P, Kacin A, Tipton MJ, Eiken O, Mekjavic IB (2004) Hypoxia increases the cutaneous threshold for the sensation of cold. Eur J Appl Physiol 92:62-68

Golja P, Mekjavic IB (2003) Effect of hypoxia on preferred hand temperature. Aviat Space Environ Med 74:522-526

Hoitsma E, Drent M, Verstraete E, Faber CG, Troost J, Spaans F, Reulen JPH (2003) Abnormal warm and cold sensation thresholds suggestive of small-fibre neuropathy in sarcoidosis. Clin Neurophysiol 114:2326-2333

Johnston CE, White MD, Wu M, Bristow GK, Giesbrecht GG (1996) Eucapnic hypoxia lowers human cold thermoregulatory response thresholds and accelerates core cooling. J Appl Physiol 80:422-429

Katayama K, Sato K, Matsuo H, Ishida K, Iwasaki K,Miyamura M (2004) Effect of intermittent hypoxia on oxygen uptake during submaximal exercise in endurance athletes. Eur J Appl Physiol 92:75-83

Kemler MA, Reulen JPH, van Kleef M, Barendse GAM, van den Wildenberg FAJM, Spaans F (2000) Thermal thresholds in complex regional pain syndrome type I: sensitivity and repeatability of the methods of limits and levels. Clin Neurophysiol 111:1561-1568
Kennedy RS, Dunlap WP, Banderet LE, Smith MG, Houston CS (1989) Cognitive performance deficits in a simulated climb of Mount Everest: Operation Everest II. Aviat Space Environ Med 60:99-104

Launay JC, Besnard Y, Guinet-Lebreton A, Savourey G (2006) Acclimation to intermittent hypobaric hypoxia modifies responses to cold at sea level. Aviat Space Environ Med 77:1230-1235

Mackintosh JH, Thomas DJ, Olive JE, Chesner IM, Knight RJ (1988) The effect of altitude on tests of reaction time and alertness. Aviat Space Environ Med 59:246-248

Mitchell D, Wyndham CH (1969) Comparison of weighting formulas for calculating mean skin temperature. J Appl Physiol 26:616622

Ramanathan NL (1964) A new weighing system for mean surface temperature of the human body. J Appl Physiol 19:531-533

Reulen JP, Lansbergen MD, Verstraete E, Spaans F (2003) Comparison of thermal threshold tests to assess small nerve fiber function: limits vs. levels. Clin Neurophysiol 114:556-563

van Marken Lichtenbelt WD, Frijns AJ, van Ooijen MJ, Fiala D, Kester AM, van Steenhoven AA (2007) Validation of an individualised model of human thermoregulation for predicting responses to cold air. Int J Biometeorol 51:169-179

Wilber RL (2001) Current trends in altitude training. Sports Med 31:249-265

Yarnitsky D, Sprecher E (1994) Thermal testing: normative data and repeatability for various test algorithms. J Neurol Sci 125:39-45 